\title{
Modelling of the water retention characteristic of deformable soils
}

\author{
Yu Wang ${ }^{1, a}$, Daichao Sheng ${ }^{2}$, Keith Ross ${ }^{1}$, David G. Toll ${ }^{3}$ \\ ${ }^{1}$ School of Computing, Science \& Engineering, University of Salford, Manchester M5 4WT, UK \\ ${ }^{2}$ Centre for Geotechnical and Materials Modeling, University of Newcastle, NSW 2308, Australia \\ ${ }^{3}$ School of Engineering, University of Durham, UK
}

\begin{abstract}
A recently proposed water retention model has been further developed for the application on unsaturated deformable soils. The physical mechanisms underpinning the water retention characteristic of soils was at first described in terms of traditional theories of capillarity and interfacial physical chemistry at pore level. Then upscaling to macroscopic level of material scale in terms of average volume theorem produces an analytical formula for the water retention characteristic. The methodology produces an explicit form of the water retention curve as a function of three state parameters: the suction, the degree-of-water-saturation and the void-ratio. At last, the model has been tested using experimental measurements.
\end{abstract}

\section{Introduction}

In geotechnical engineering applications, it has been widely accepted that the water retention characteristic (WRC) of deformable soils should explicitly take into account of the coupling effects of the hydraulic and mechanical behaviours [1], such as the volume change or the void ratio change in soils as a function of the water content or the variation of suction, or both of these effects. As a result, the water retention characteristic of deformable soils presents a curve in the 3D space of suction/water-content/void-ratio [2] or in the equivalent space of suction/the-degree-of-water-saturation/specificvolume [3]. In addition, for any particular deformable soil, its water retention characteristic also depends on it initial state, particular its initial porosity or void ratio. This fact means that the water retention characteristic of a deformable soil presents a surface which is called the soil-water retention surface (SWRS) [2], and any specific water retention curve of the soil should in the surface. Many researchers have contributed to the description of the SWRS of deformable soils. Gallipoli et al. [3] suggested to take one of the parameters in the original van Genuchten [4] formula to be a function of the specific volume. Stange \& Horn [5] assumed that the parameters in the original van Genuchten formula depend on the void ratio. Tarantino [6] proposed a power function for the suction in terms of the water ratio and combined this with the original van Genuchten formula. Wheeler et al. [7] used a bilinear function between the degree of saturation and the logarithmic modified suction. Mbonimpa et al. [8] proposed a modified Kovacs (MK) model which incorporates the void ratio into the relationship between the degree of water saturation and the suction. Marsin [9] used a suction rate as a function of

\footnotetext{
a Corresponding author: y.wang@salford.ac.uk
}

the deformation rate. Sheng \& Zhou [10] suggested a general differential form of the relationship between the degree of water saturation and suction and voids ratio. In addition to these empirical approaches, a fractal method has also been adopted [11,12].

In hydrology and soil physics, much progress has been made adopting the approach based on fundamental physical description. Assouline et al. [13] proposed a conceptual model to relate the pore volume to the particle volumes in terms of a power function, then adopt the capillary equation to define the water retention curve. Taking account of the effect of the water film on empty pore surfaces, Tuller et al. [14] proposed a unit cell model for water retention characteristic modeling [15]. Chertkov [16-18] proposed a physically based model to define the suction of soil matrix as a product of two physical factors which stand for the adsorption-capillary effect and the influence of variation of soil-porosity, respectively. Generally, these models describe WRC in the plane of water saturation and suction $\left(S_{w}-s\right)$.

Wang et al. [19] proposed a physical-chemical WRC model based on the classical theory of capillarity, interfacial physical chemistry and the average volume theorem. This paper is to investigate the application of the model on deformable unsaturated soils.

\section{A Water Retention Model}

From the point view of interfacial physical chemistry, a fluid in a porous medium can be regarded as being adsorbed by the pore surface [20], where the fluid is called as the adsorbate and the pore surface is called the substrate which exerts adsorptive forces on the adsorbate due to atom and molecular interaction at the interfacial 
region [21]. The adsorptive forces will modify the chemical potential of the molecules of the adsorbate at the interfacial region in the reference to the state when the bulk adsorbate fluid is under a free and open atmospheric condition [22]. In general, the chemical potential change depends on the distance of the molecule of the adsorbate above the surface of the substrate [14]. In unsaturated soils, the water and vapour phases have different individual pressures due to their different chemical potential changes [19]. Under the equilibrium state of a mechanical balance, the pressure difference of the two fluid phases in the pore system follows the Laplace equation [23], i.e.:

$$
p_{c}=p_{w}-p_{v}=-\frac{2 \sigma \cos \theta}{r}
$$

where $p_{c}$ is called the capillary pressure or matric potential at pore scale, and its absolute value is called as suction; $p_{w}$ and $p_{v}$ are the microscopic intrinsic pore pressures of the water and vapour phases, respectively; $\sigma$ is the surface tension on the meniscuses, $r$ indicates the pore radius at the position of meniscuses, $\theta$ is the contact angle between the meniscus and the pore surface.

The chemical potential change of the adsorbates on a substrate surface can be evaluated using the Kelvin equation [20, 14], as the result, the intrinsic pressure of the adsorbates on substrates can be expressed as [19]:

$$
p_{f}=\frac{\bar{u}_{f}}{V_{f}}+p_{0} \exp \left(\frac{\bar{u}_{f}}{R T}\right)
$$

where $p_{f}$ is the average intrinsic pressure of the fluid on the surface; $\bar{u}_{f}$ is the average intrinsic molar potential change of the fluid of a thickness $h$, which is defined as $\bar{u}_{f}=\frac{1}{h} \int_{0}^{h} u_{f}(z) d z$, where $u_{f}(\mathrm{z})$ is the fluid molar molecule potential change at position $z$ above the substrate surface; $V_{f}$ is the molar volume of the liquid phase. $R$ is the gas constant; $T$ is the temperature; $p_{0}$ is a normal pressure.

Eq. (2) consists of two terms: the first term takes account of the intrinsic chemical potential change, while the second term is it physical effect, which is called the gauge pressure to be measurable directly. Practically, the physical state of the fluid on substrates can be expressed using the gauge pressure, i.e.:

$$
p_{f}=p_{0} \exp \left(\frac{\bar{u}_{f}}{R T}\right)
$$

In terms of the mean-field model [24], Tuller et al. [14] suggested that the chemical potential change $\bar{u}_{f}$ in Eq.

(3) can be estimated in terms of the fluid film thickness $\left(t_{f}\right)$ on the surface of substrates using the following equation:

$$
\bar{u}_{f}=\frac{A^{m}}{t_{f}^{3}}+\frac{A^{e}}{t_{f}^{2}}+\frac{A^{s}}{t_{f}}
$$

where the constant $A^{m}$ relates to disjoining force due to van der Waals molecular interaction force, $A^{e}$ relates to the interfacial electrostatic effect and $A^{s}$ depends on the structure of substrate surface. In addition to the molecular interaction, in terms of the statistical molecular mechanics for the liquid-gas surface [25], the total chemical potential change of the wetting fluid film on substrate surfaces should consist of two components of the 'intrinsic' and the 'external' natures. The intrinsic component may be defined in terms of Eq. (4), while the external component depends on the other physical effects rather than molecular interfacial interactions. As an improvement, the Eq. (4) may be revised into the following form:

$$
\bar{u}_{f}=\frac{A^{m}}{t_{f}^{3}}+\frac{A^{e}}{t_{f}^{2}}+\frac{A^{s}}{t_{f}}+A^{0}
$$

where $A^{0}$ is a new constant introduced for the external effect such as the curvature of pore wall surface. Mathematically, Eq. (5) may be characterised into the following form:

$$
\bar{u}_{f}=\frac{A_{1}}{t_{f}^{b}}+A_{0}
$$

where $A_{1}, A_{0}$ and $b$ are three new constants.

For unsaturated soils, representing the pores in soils using a bundle of capillary cylinders (the BCC modelling), a complicated pore system can be equivalent to a capillary tube of a characterised 'hydraulic radius', which is defined as ratio of total pore volume to total pore surface area. Based on the characterised capillary tube, the bulk water phase in the water filled part and the coexisting vapour phases in the empty part will have their respective chemical potential changes and physical pressures, which can be described using the Eq. (6) and (3). For example, for the water phase, its chemical potential change can be expressed as:

$$
\begin{aligned}
\Delta \bar{u}_{w} & =\frac{1}{A_{S}} \int_{A_{w}} \bar{u}_{w} d A \\
& =\frac{1}{A_{S}} \int_{A_{w}}\left(\frac{A_{1, w}}{r_{h}^{b_{w}}}+A_{0, w}\right) d A \\
& =\left(\frac{A_{1, w}}{r_{h}^{b_{w}}}+A_{0, w}\right) \frac{A_{w}}{A_{S}}+\bar{u}_{0} \\
& =\bar{u}_{w} S_{w}+\bar{u}_{0}
\end{aligned}
$$

where $r_{h}$ is the radius of the capillary tube or the hydraulic radius of the porous medium. $A_{1, w}, A_{0, w}$ and $b_{w}$ are three constants depending on the nature of the material and the hydraulic radius of the porous medium. $A_{S}$ is the total surface area of the tube or the pores of the porous media represented. $A_{w}$ is the area of the part of the surface occupied by the bulk water phase. As a result, $\bar{u}_{w}=\frac{A_{1, w}}{r_{h}^{b}}+A_{0, w}$ is the local average of the chemical potential change along the radius of the capillary tube. $S_{w}$ is the degree of water saturation or the fraction of the volume of the capillary tube occupied by the bulk water 
phase. $\bar{u}_{0}$ is the initial chemical potential change when bulk water starts to fill the tube due to capillary condensation, which depends on the initial water molecular film on the totally empty pore surface [26]. Substituting the Eq. (7) into (3) for $\bar{u}_{f}$ produces the following Eq. (8) which describes the pressure of the bulk water in the tube in terms of the water saturation.

$$
\begin{aligned}
p_{w} & =p_{0} \exp \left(\frac{\Delta \bar{u}_{w}}{R T}\right)=p_{0} \exp \left(\frac{\bar{u}_{w} S_{w}+\bar{u}_{0}}{R T}\right) \\
& =p_{0} \exp \left(\frac{\bar{u}_{0}}{R T}\right) \exp \left(\alpha S_{w}\right)
\end{aligned}
$$

where $\alpha=\frac{\bar{u}_{w}}{R T}$. A similar approach may be applied on the coexisting vapour phase in unsaturated soils, and the vapour pressure can be estimated in terms of the degree of water saturation as:

$$
p_{v}=p_{0} \exp \left(\frac{\bar{u}_{0}}{R T}\right) \exp \left(\beta\left(1-S_{w}\right)\right)
$$

where $\beta=\frac{\bar{u}_{v}}{R T}$ and $\bar{u}_{v}=\frac{A_{1, v}}{r_{h}^{b_{v}}}+A_{0, v}$ is a local average of the chemical potential change of the vapour phase along the radius of the capillary tube. $A_{1, v}, A_{0, v}$ and $b_{v}$ are taken as three constants.

Following the volume average theorem [27, 28], the Darcy-scale capillary pressure can be expressed as:

$$
\begin{aligned}
& \left\langle p_{c}\right\rangle=\left\langle p_{w}\right\rangle-\left\langle p_{v}\right\rangle= \\
& \frac{1}{V_{R E V}} \int_{V_{w}} p_{w} d V-\frac{1}{V_{R E V}} \int_{V_{v}} p_{v} d V
\end{aligned}
$$

where $\left\langle p_{c}\right\rangle$ indicates the macroscopic volume average of the capillary pressure. Substituting Eqs. (8) and (9) into (10) for $p_{w}$ and $p_{v}$, respectively, generates the following definition of the average capillary pressure in terms of the degree of water saturation [29]:

$$
\left\langle p_{c}\right\rangle=\lambda\left[\frac{1}{\beta}-\frac{1}{\alpha}+\frac{1}{\alpha} \exp \left(\alpha S_{w}\right)-\frac{1}{\beta} \exp \left(\beta\left(1-S_{w}\right)\right)\right]
$$

where, $\lambda=\varepsilon p_{0} \exp \left(\frac{\bar{u}_{0}}{R T}\right), \varepsilon=V_{\text {pore }} / V_{\text {bulk }}$ is the porosity.

\section{Modelling Water Retention Characteristic of Deformable Soils}

Using the Eq. (11) to describe the SWRS in the 3D space, we need to know the intrinsic relationship between the three parameters $(\lambda, \alpha$ and $\beta)$ and the change of pore structure (porosity or void ratio). According to Eqs. (7)(9), $\alpha$ and $\beta$ depend on the characterised hydraulic radius, $r_{h}=\frac{V_{\text {pore }}}{A_{S}}$. Assuming the total pore surface area $A_{S}$ and the total volume of solid phase $V_{\text {solid }}$ do not change, we can have $r_{h}=\frac{V_{\text {solid }}}{A_{S}} \times \frac{V_{\text {pore }}}{V_{\text {solid }}}=C \cdot e$, where $C$ is a constant and $e=V_{\text {pore }} / V_{\text {solid }}$ is the void ratio. Taking the intrinsic relationships that porosity $\varepsilon=\frac{e}{1+e}$ and water saturation $S_{w}=\frac{w}{e}$, where $w=V_{\text {water }} / V_{\text {solid }}$ is water ratio, Eq. (11) can be rewritten into the following form:

$$
\left\langle p_{c}\right\rangle=\frac{\lambda e}{1+e}\left[\begin{array}{l}
\frac{1}{\frac{B_{2}}{e^{B_{3}}}+B_{1}}-\frac{1}{\frac{A_{2}}{e^{A_{3}}}+A_{1}}+\frac{1}{\frac{A_{2}}{e^{A_{3}}}+A_{1}} \exp \left(\left(\frac{A_{2}}{e^{A_{3}}}+A_{1}\right) \frac{w}{e}\right)- \\
\frac{1}{\frac{B_{2}}{e^{B_{3}}+B_{1}}} \exp \left(\left(\frac{B_{2}}{e^{B_{3}}}+B_{1}\right)\left(1-\frac{w}{e}\right)\right)
\end{array}\right]
$$

where the parameters $\lambda, A_{i} \mathrm{~s}$ and $B_{i} \mathrm{~s}$ are redefined by merging with other constants. $A_{i}$ s relate to the bulk water phase in pore spaces, while $B_{i}$ s relate to the coexisting vapour phase in pore spaces. the term $R T$ has been taken into $A_{i} \mathrm{~s}$ and $B_{i} \mathrm{~s}$. In the next section, the Eq. (12) is used to model the SWRS of three unsaturated soils reported in references by fitting all of the measured residual shrinkage SWRCs of each soil in the space of $s-w-e$.

\section{Modelling Examples and Discussion}

In this paper, the experimental data are taken from previously reported experiments on the deformable soils under draining hydraulic loadings only. Three series of experimental data of soil under draining shrinkage are chosen from the published references. They are two sets of undisturbed samples from a field site and one set of samples reconstituted in laboratory to different porosities. The modelling of each set of samples is conducted in the space of matric suction, water ratio and void ratio $(s-w-e)$ for all of the measured shrinking curves of different initial void ratios.

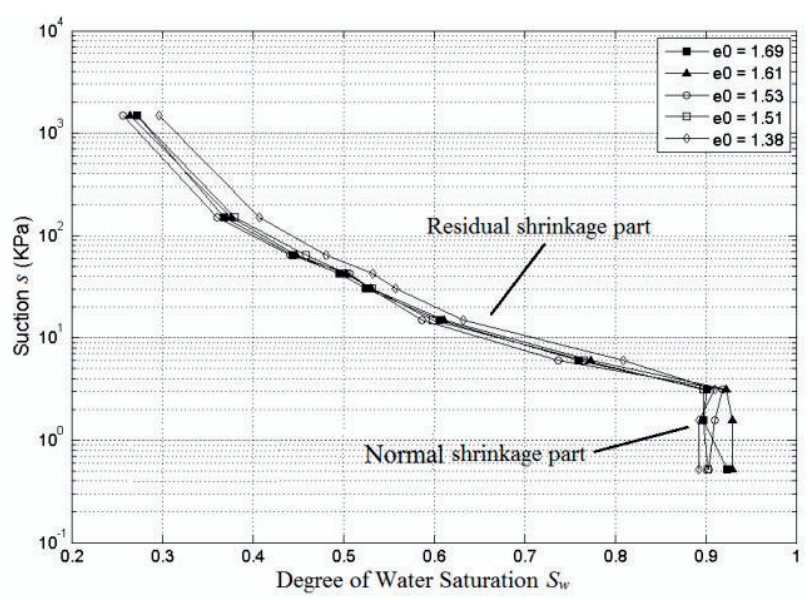

Figure 1. The plot, in $S_{w}-s$ plane, of the SWRCs of the Stagnic Chernozem soil samples at $A_{p}$ horizons $(0-30 \mathrm{~cm})$ [5] 


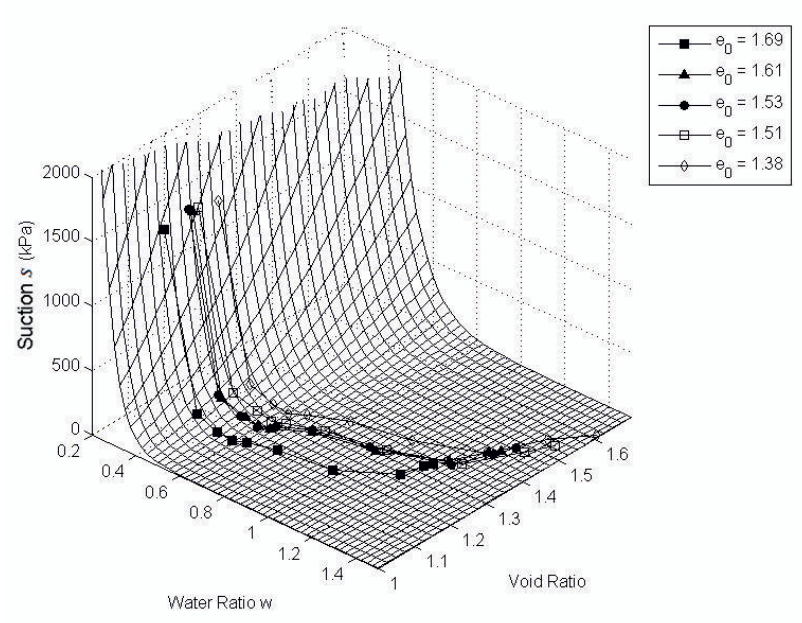

Figure 2. The modelling result for the SWRS of $A_{p}$
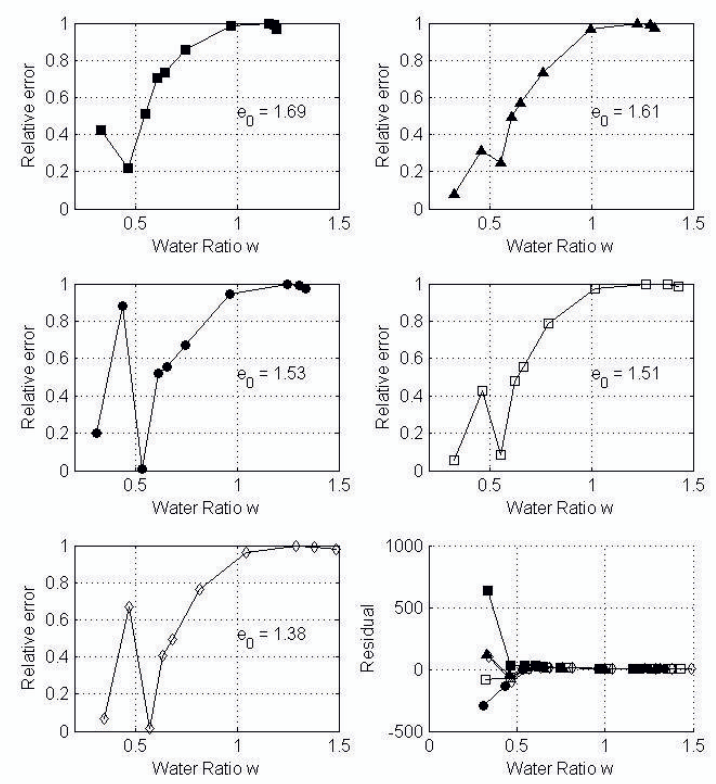

Figure 3. The modelling relative errors and residuals for the individual SWRCs in Fig. 2

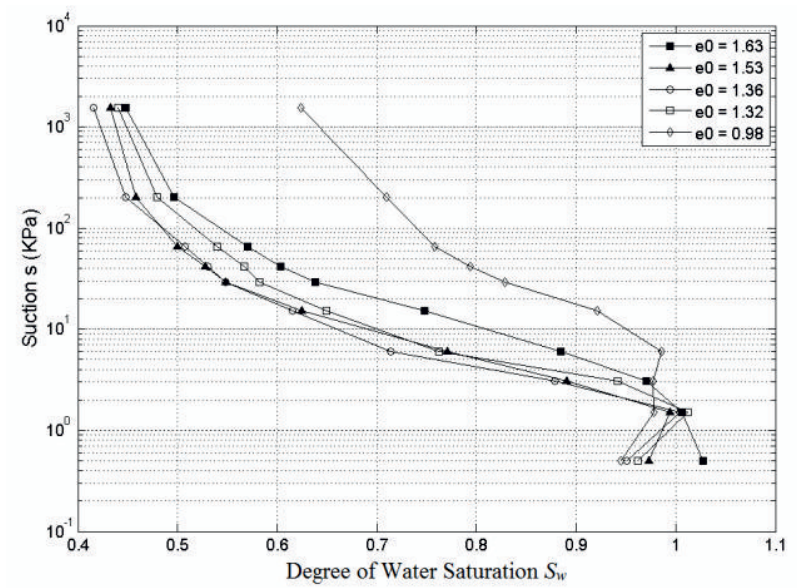

Figure 4. The plot, in $S_{w}-s$ plane, of the SWRCs of the Stagnic Chernozem soil samples at $\mathrm{A}_{\mathrm{h}}$ horizons (30$50 \mathrm{~cm})[5]$
Stange and Horn [5] reported an experiment (Fig. 1) on a deformable soil which was taken from a site called Stagnic Chernozem in Germany. The soil samples of different initial void ratios, $\mathrm{e}_{0}$, were from a shallow range of horizons $A_{p}(0-30 \mathrm{~cm})$. They were exposed to a series of desaturation to different pressure head/suction values without any external mechanical loading except for the self-gravimetric effect. The SWRCs in Fig. 1 have displayed two distinct parts which represent two types of deformation, i.e. the normal shrinkage part, where saturation has little change, when suction $s<3.1 \mathrm{kPa}$ or the degree of water saturation $S_{w} \cong 0.9$ and the residual shrinkage part elsewhere $\left(S_{w}<0.9\right)$. It can be seen that, in the normal shrinkage part, the degree of water saturation $S_{w}$ changes little while the void ratio $e$ of the soil samples decreases. This normal shrinkage part is known as the consolidation phase of soils. It also shows that the air entry value of the suction also changes little in the range of the initial void ratio $\mathrm{e}_{0}$ - from 1.38 to 1.96 . Because Eq. (12) derives from the original form of Eq. (11) for the relation of the matric suction and the degree of water saturation, it does not work for the normal shrinkage part. So it is only used to model the residual shrinkage part in this paper.

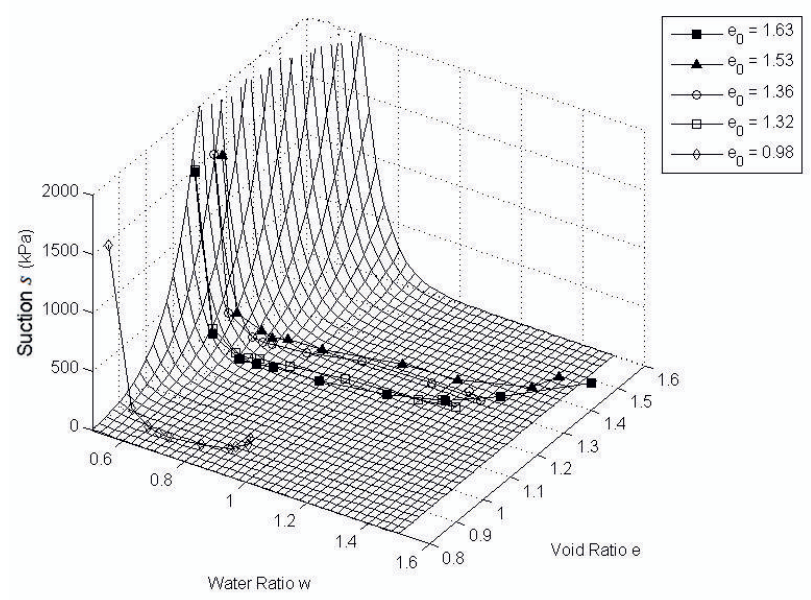

Figure 5. The modelling result for the SWRS of $A_{h}$

Fig. 2 replots these curves in the 3D space of $s-w-e$ while shows the predicted SWRS result using the Eq. (12) to fit all of these curves. The corresponding fitting data are listed in Table 1. Fig. 3 shows the relative errors $\left(\frac{\left|F_{i}-f_{i}\right|}{f_{i}}\right)$ and the residuals $\left(F_{i}-f_{i}\right)$ of the predicted suction to the measurements, where $F_{i}$ is the modelling result at the measured point $i$, and $f_{i}$ is the measurement at that point. Both Figs. 2 and 3 have shown that Eq. (12) gives a reasonably good modelling result. The predicted SWRS fits all of the measured SWRCs, in the whole range of the variation of $s, w$ and $e$, reasonably well in terms of the shape and the calculated errors. In terms of the residual in Fig. 3, a non-ideal point presents at the highest suction or the lowest water ratio of the curve of $\mathrm{e}_{0}$ $=1.69$. The worst accuracy at the point may relate to two reasons. Firstly, the accurate point of the highest suction corresponding to the lowest water content is very hard to be precisely measured. Secondly, because the Eq. (12) 
takes an assumption of a non-changed pore surface area, in the case if the soil skeleton collapses due to relatively slide or relocation of particles, the collapse of pore structure could cause new blockages or inaccessible pores that will change the total pore surface areas taken into account by Eq. (12). When samples are under high suction and of low water content, the collapse of pore structure is highly possible.

Fig. 4 shows the measured SWRCs of another set of samples which were taken from a relatively deep range of horizons $A_{h}(30-50 \mathrm{~cm})$ of the Stagnic Chernozem soil [5]. Fig. 5 shows the predicted SWRS result using the Eq. (12) to fit these curves in the 3D space of $s-w-e$. The corresponding fitting data are listed in Table 1. The calculated relative errors and residuals of the modelling suction results against the measurements (Fig. 6) have also showed that the Eq. (12) produces a reasonably good modelling for most of these curves. It can be seen that the curve of the soil sample $e_{0}=0.98$ presents an inconsistence with the others. Noticed that its initial porosity is much smaller than that of the others, it is expected to possess a distinctively different pore structure of significantly different pore surface area.
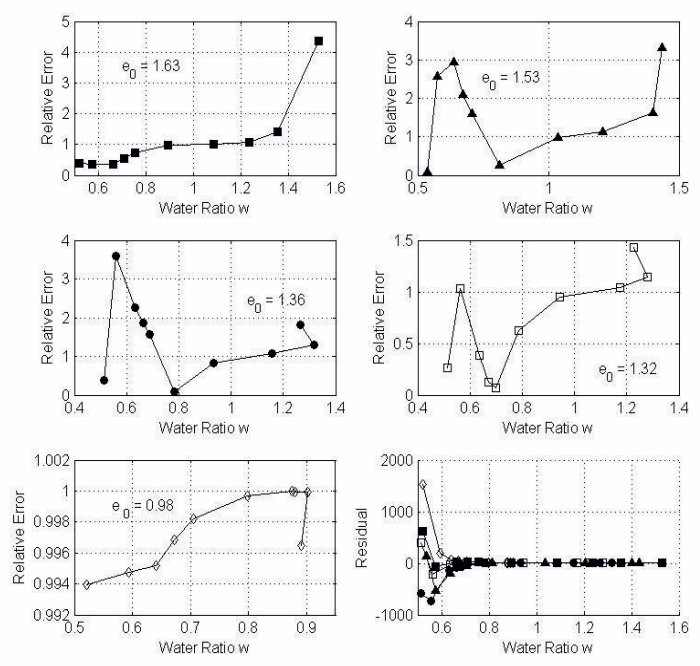

Figure 6. The modelling relative errors and residuals for the individual SWRCs in Fig. 5

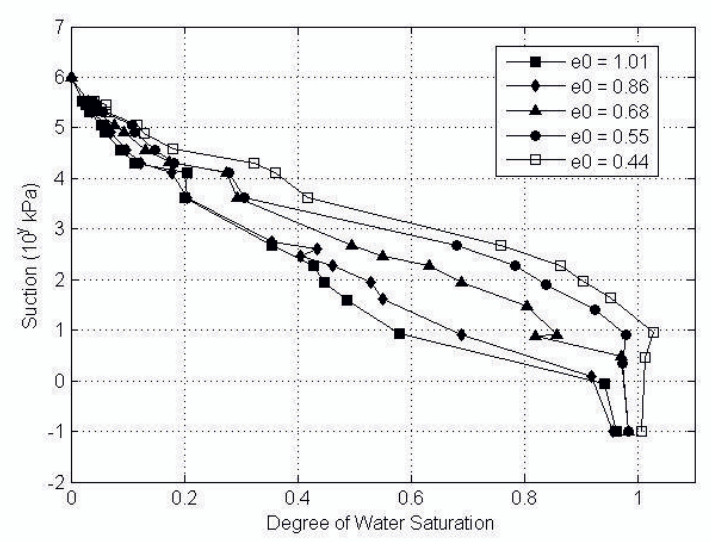

Figure 7. The plot, in $S_{w}-s$ plane, of the SWRCs of a clayey-silty-sand soil samples under a desaturation process [2]
Salager et al. [2] reported an experiment (Fig. 7) and modelling of the SWRS for a reconstituted soil, which consists of clay $(10 \%)$, silt $(18 \%)$ and sand $(72 \%)$. Fig. 8 shows the SWRS modelling results using the Eq. (12) to fit all of the SWRC measurements in the space of $s-w-e$. The relative errors and residuals of the modelling result against to the experimental data have showed that a reasonably good predicting result has been obtained again. For each curve, the relative error (Fig. 9) at the low water content (high suction) is lower than that at high water content (low suction), but the residual at low water content is higher than that at high water content. This is because of that the magnitude of suction is much greater at low water contents.

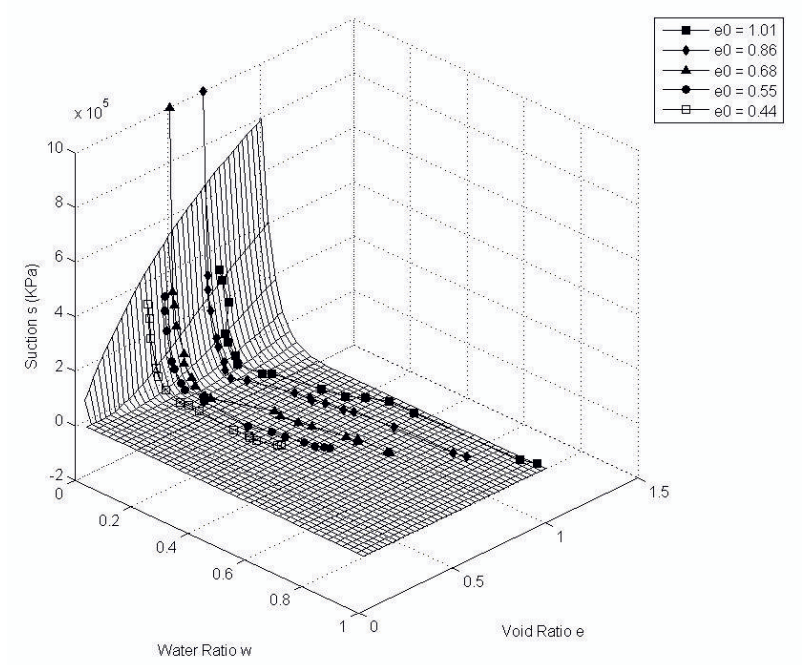

Figure 8. The modelling result for the SWRS of a clayey-silty-sand soil [2]
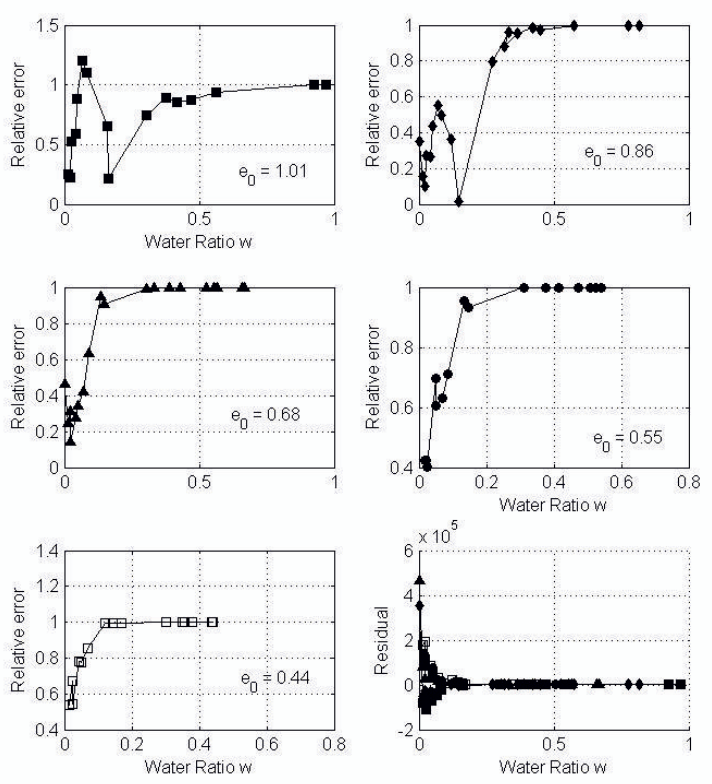

Figure 9. The modelling relative errors and residuals for the individual SWRCs in Fig. 8

From all the modelling examples we can see that, in terms of the relative error, the model has a better 
performance in high suction region than in low suction region. This is explained due to the modelling of the vapour phase, where we neglect the effect of the wetting water film on the wall of empty pores, which increases with the increase of the degree of water saturation or vice versa, and take the parameter, $\beta$, as constant for the vapour phase.

In the current work, the water film has been effectively treated with the bulked condensed capillary water together. If the film thickness is very small such as at high suctions, the effect of the water film will not be significant. Iwamatsu and Horii [24] have demonstrated that the capillary condensation radius is more than one order bigger than the wetting film thickness). However, at low suctions, particularly when the pore radius is bigger than that of significant capillary condensation effect, such the effective treatment of the water film brings in the deterioration of accuracy.

Table 1. The fitting parameters data using Eq. (12)

\begin{tabular}{|c|c|c|c|}
\hline Samples & $\mathrm{A}_{\mathrm{h}}$ & $\mathrm{A}_{\mathrm{p}}$ & $\begin{array}{c}\text { clayey-silty- } \\
\text { sand soil }\end{array}$ \\
\hline$\lambda$ & -0.03927 & -0.09463 & $-8.658 \mathrm{e}-4$ \\
\hline$A_{1}$ & 6.581 & 225 & $1.392 \mathrm{e}-2$ \\
\hline$A_{2}$ & -25.39 & -1794 & -1611 \\
\hline$A_{3}$ & 9.107 & 0.4636 & 233.4 \\
\hline$B_{1}$ & 25.2 & 17.97 & 24.53 \\
\hline$B_{2}$ & $-1.49 \mathrm{e}-3$ & $-8.304 \mathrm{e}-3$ & $-2.855 \mathrm{e}-4$ \\
\hline$B_{3}$ & $6.271 \mathrm{e}-5$ & $1.532 \mathrm{e}-3$ & $1.186 \mathrm{e}-2$ \\
\hline
\end{tabular}

\section{Conclusions}

This paper reports a model of the water retention characteristic of unsaturated porous media and its application on the residual shrinkage of deformable soils. The model is based on an equation of the state of the fluid in porous media established on the classical capillary theory. It explicitly take account of the effect of deformation and can be used to fit the measured SWRCs of different initial void ratios of a soil and constructs a general SWRS in the space of $s-w-e$. The tests on three soils have shown that the model works well in terms of the shape of the modelled SWRS and the calculated relative errors and residuals for modelling accuracy. The explanation on its performance is in agreement with the fundamental concepts and the assumptions of the model. By now, the model has been proved to work particularly well for high suction region when the capillary condensation plays a significant role. A further work to improve its performance at low suction region needs the consideration of the effect of the wetting water film on the empty pore surface. On another hand, it needs to be pointed out as well that modelling the water retention characteristic of deformable soils in terms of the soilwater retention surface is not enough. Further investigation in future needs to be able to explicitly describe each of the specific water retention curves of different initial void ratios in the space of $s-w-e$.

\section{References}

1. D. Sheng, Computers and Geotechnics, 38, (2011)

2. S. Salager, M.S. EI Youssoufi, C. Saix, Can. Geotech. J. 47, (2010)

3. D. Gallipoli, S.J. Wheeler, M. Karstunen, Geotechnique 53, (2003)

4. M.T. van Genuchten, Soil Science Society of America Journal 44, (1980)

5. F. Stange, R. Horn, Vadose Zone Journal, 4, (2005)

6. A.Tarantino, Geotechnique, 59, (2009)

7. S.J. Wheeler, S.R. Sharma, R.S.R. Buisson, 2003, Geotechnique 53, (2003)

8. M. Mbonimpa, M. Aubertin, A. Maqsoud, B. Bussiere, Journal of Geotechnical and Geoenvironmental Engineering 132, (2006)

9. D. Masin, International Journal for Numerical and Analytical Methods in Geomechanics 34, (2010)

10. D. Sheng, A.N. Zhou, Canadian Geotechnical Journal 48, (2011)

11. A.R. Russell, European Journal of Soil Science 61, (2010)

12. N.R.A. Bird, E. Perrier, M. Rieu, European Journal of Soil Science 51, (2000)

13. S. Assouline, D. Tessier, A. Bruand, Water Resources Research 34, (1998)

14. M. Tuller, D. Or, L.M. Dudley, Water Resources Research 35, (1999)

15. D. Or, M. Tuller, Water Resources Research 35, (1999)

16. V.Y. Chertkov, Journal of Hydrology 286, (2004)

17. V.Y. Chertkov, 2010, The Open Hydrology Journal 4, (2010)

18. V.Y. Chertkov, Advances in Water Resources 44, (2012)

19. Y. Wang, S.M. Grove, M.G. Anderson, Advances in Water Resources 31, (2008)

20. N.V. Churaev, Liquid and Vapour Flow in Porous Bodies: Surface phenomena, (Gordon and Beach Science Publishers, Amsterdam, 2000)

21. A.W. Adamson, Physical chemistry of surfaces (John Wiley \& Sons, 1982)

22. F. Rouquerol, J. Rouquerol, K. Sing, Adsorption by Powers \& Porous Solids - Principles, Methodology and Applications (Academic Press, London, 1999)

23. F.A.L. Dullien, Porous media fluid transport and pore structure, (Academic Press Inc., USA, 1991)

24. M. Iwamatsu, K. Horii, Journal of Colloid Interface Science 182, 1996

25. J.S. Rowlinson, B. Widom, Molecular Theory of Capillarity, (Oxford University Press, NY, 1984)

26. Y. Wang, Journal of Porous Media 13, (2010)

27. J.M. Nordbotten, M.A. Celia, H.K. Dahle, S.M. Hassanizadeh, Water Res. Res. 43, (2007)

28. C.L. Tucker III, R.B. Dessenberger, Flow and rheology in polymer composites manufacturing (Elsevier Science, 1994)

29. Y. Wang, X.Y. Wang, M. Scholz, D.K. Ross, Cons and Built Mats 35, (2012) 